\title{
Selective constraint acting on TLR2 and TLR4 genes of Japanese Rana frogs
}

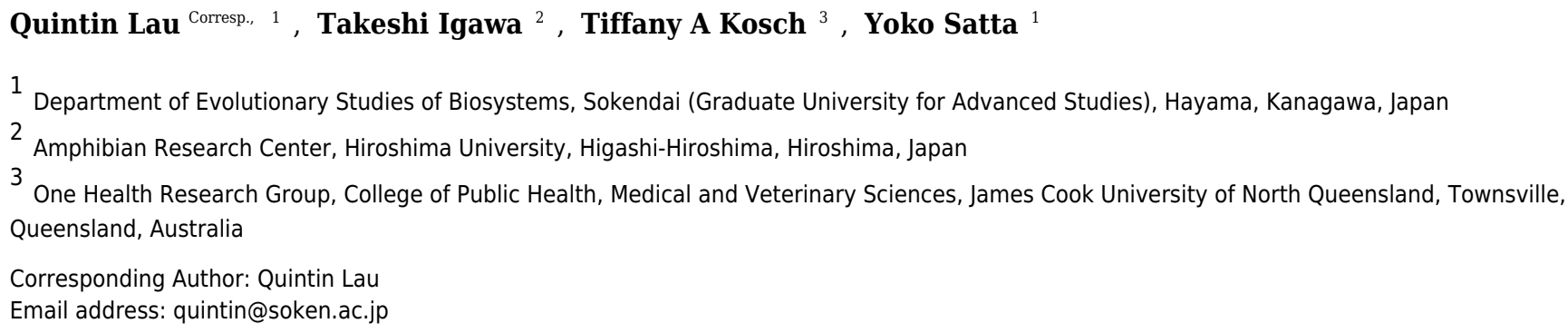

Toll like receptors (TLRs) are an important component of innate immunity, the first line of pathogen defence. One of the major roles of TLRs includes recognition of pathogenassociated molecular patterns. Amphibians are currently facing population declines and even extinction due to chytridiomycosis caused by the Batrachochytrium dendrobatidis (Bd) fungus. Evidence from other vertebrates shows that TLR2 and TLR4 are involved in innate immunity against various fungi. Such genes therefore may play a functional role in amphibian-chytridiomycosis dynamics. Frogs from East Asia appear to be tolerant to Bd, so we examined the genetic diversity that underlies TLR2 and TLR4 from three Japanese Ranidae frog species, Rana japonica, $R$. ornativentris and $R$. tagoi tagoi ( $\mathrm{n}=5$ per species). We isolated 27 TLR2 and 20 TLR4 alleles and found that these genes are evolutionarily conserved, with overall evidence supporting purifying selection. In contrast, site-by-site analysis of selection identified several specific codon sites under positive selection, some of which were located in the variable leucine rich repeat domains. In addition, preliminary expression levels of TLR2 and TLR4 from transcriptome data showed overall low expression. Although it remains unclear whether infectious pathogens are a selective force acting on TLRs of Japanese frogs, our results support that certain sites in TLRs of these species may have experienced pathogen-mediated selection. 


\section{Selective constraint acting on TLR2 and TLR4 genes of Japanese Rana frogs.}

2 Quintin Lau ${ }^{1}$, Takeshi Igawa ${ }^{2}$, Tiffany A. Kosch ${ }^{3}$, Yoko Satta ${ }^{1}$

3 'Department of Evolutionary Studies of Biosystems, Sokendai (The Graduate University for Advanced

4 Studies), Kamiyamaguchi 1560-35, Hayama, Kanagawa 240-0193, Japan.

$5{ }^{2}$ Amphibian Research Center, Hiroshima University, 1-3-1, Higashi-Hiroshima, Hiroshima 739-8526, 6 Japan.

7 3One Health Research Group, College of Public Health, Medical and Veterinary Sciences, James Cook

8 University, Townsville, Queensland, 4811, Australia.

9 Corresponding author: Quintin Lau, email: quintin@soken.ac.jp , Tel: +81 46858 1610, Fax: +81 46858

$10 \quad 1544$

11 Takeshi Igawa, email: tigawa@hiroshima-u.ac.jp

12 Tiffany A Kosch, email: tiffany.kosch@jcu.edu.au

13 Yoko Satta, email: satta@soken.ac.jp 


\section{Abstract}

15 Toll like receptors (TLRs) are an important component of innate immunity, the first line of pathogen

16 defence. One of the major roles of TLRs includes recognition of pathogen-associated molecular patterns.

17 Amphibians are currently facing population declines and even extinction due to chytridiomycosis caused

18 by the Batrachochytrium dendrobatidis $(\mathrm{Bd})$ fungus. Evidence from other vertebrates shows that TLR2

19 and TLR4 are involved in innate immunity against various fungi. Such genes therefore may play a

20 functional role in amphibian-chytridiomycosis dynamics. Frogs from East Asia appear to be tolerant to

$21 \mathrm{Bd}$, so we examined the genetic diversity that underlies TLR2 and TLR4 from three Japanese Ranidae

22 frog species, Rana japonica, $R$. ornativentris and $R$. tagoi tagoi ( $\mathrm{n}=5$ per species). We isolated 27 TLR2

23 and 20 TLR4 alleles and found that these genes are evolutionarily conserved, with overall evidence

24 supporting purifying selection. In contrast, site-by-site analysis of selection identified several specific

25 codon sites under positive selection, some of which were located in the variable leucine rich repeat

26 domains. In addition, preliminary expression levels of TLR2 and TLR4 from transcriptome data showed

27 overall low expression. Although it remains unclear whether infectious pathogens are a selective force

28 acting on TLRs of Japanese frogs, our results support that certain sites in TLRs of these species may have

29 experienced pathogen-mediated selection. 


\section{Introduction}

32

33

Toll-like receptors (TLRs) are a type of pattern recognition receptor that recognize pathogen-associated molecular patterns (PAMPs) such as bacterial cell walls and nucleic acids (Medzhitov, 2001). The signalling of TLRs triggers the synthesis and release of pro-inflammatory cytokines, and thus TLRs have an important role in innate immunity and activation of adaptive immunity. TLRs are type 1 membrane glycoproteins comprised of extracellular and cytoplasmic domains; the extracellular domain is also considered as a 'pathogenrecognition domain' with a variable number of leucine rich repeats (LRR, protein motifs in the ectodomain inferred to be important for recognising molecules), while the cytoplasmic signalling domain is a conserved toll/IL-1 domain (Mikami et al., 2012). The TLR repertoire differs between vertebrate groups ranging from 10 loci in humans and great apes to 21 in urodele amphibians (Takeda, Kaisho \& Akira, 2003; Quach et al., 2013; Babik et al., 2014). Specific TLR loci are generally considered to recognize different groups of PAMPs, for example, TLR1 and TLR6 associate with TLR2 to recognize acylated lipopeptides, TLR4 recognize lipolysaccharides in gram-negative bacteria, and TLR5 recognize bacterial flagellin (Poltorak et al., 1998; Voogdt et al., 2016). In addition, TLR2 and TLR4 can recognize PAMPs derived from different fungi species (reviewed by Roeder et al., 2004; Luther \& Ebel, 2006).

In amphibians, TLRs have been described in Xenopus frogs (20 loci from 14 families, Ishii et al., 2007) and newts (21 loci from 13 families, Babik et al., 2014). TLR families characterized in both frogs and newts include TLR01, TLR02, TLR03, TLR05, TLR07, TLR08, TLR09, TLR12, TLR13, TLR14, TLR21, and TLR22. However, the prevalence of TLR4 across amphibian taxa is uncertain: no TLR4 orthologs were found in newts, but putative TLR4 were identified in Xenopus (Ishii et al., 2007; Babik et al., 2014). In addition, TLR4 was one of the 11 TLR genes that were isolated from transcriptome data of Bombina maxima frogs (Zhao et al., 2014).

Most TLR genes are functionally constrained, and typically have high sequence conservation and slow evolutionary rates to maintain a functional role of recognizing conserved PAMPs (Roach et al., 2005), although extracellular LRR domains have higher evolutionary rates compared to intracellular domains 
55 (Mikami et al., 2012). An overall signature of purifying selection was identified in TLRs of urodele amphibians, 56 although a few individual codons were found to be evolving under positive selection (Babik et al., 2014), as

57 has been observed in other vertebrates (Wlasiuk \& Nachman, 2010; Shang et al., 2018). The identification of

58 TLR-disease associations in vertebrates (Tschirren et al., 2013; Noreen \& Arshad, 2015) also supports that

59 episodic selection can occur in TLR genes as a response to changes in pathogen diversity. In addition, human

60 TLR4 displayed significantly negative Tajima's D values in nonsynonymous variants (Smirnova et al., 2001),

61 and subsequent evidence supporting selection for rare TLR4 variants was found (Smirnova et al., 2003).

62 Therefore, while TLRs may be under functional constraints, there is evidence across vertebrates that positive selection may act on these genes in response to local pathogens.

Chytridiomycosis is a disease in amphibians caused by the fungal pathogen Batrachochytridium dendrobatidis

(Bd). This disease has been linked to the decline of amphibian populations worldwide (Daszak, Cunningham \& prevalent in Korea and Japan (Goka et al., 2009; Bataille et al., 2013), within endemic East Asian frogs there is no evidence of Bd-related declines and no published reports of Bd susceptibility following experimental infection; this supports that such frogs could be Bd-tolerant. Additionally, genetic evidence for high Bd genetic diversity and endemism in this region indicates that Bd is endemic to Asia (Fisher, 2009; Bataille et al., 2013), suggesting a long co-evolutionary history between the Bd pathogen and Asian amphibians. chytridiomycosis (Lau et al., 2016, 2017), there are limited studies involving innate immunity genes including TLRs. Since TLR2 and TLR4 have been shown to play a role in innate immune responses to various fungi

75 (Roeder et al., 2004; Luther \& Ebel, 2006), TLRs therefore may be involved in Bd resistance (Richmond et al., 76 2009). a few anuran species including Bombina and Xenopus frogs. In B. maxima, expression levels of TLR2 and TLR4, 
measured using with quantitative RT-PCR, were variable between different adult tissues (Zhao et al., 2014). In

X. laevis, ubiquitous expression of both TLR2 and TLR4 was detected in adults and tadpoles using standard

81 PCR, but expression levels were not quantified (Ishii et al., 2007). This current study provides the opportunity

82 to expand the knowledge about basal TLR expression in amphibians.

83 To better understand the gene complements of anuran innate immunity, here we characterize the genetic

84 diversity and selection patterns of two candidate TLR genes in three Japanese Rana species using molecular

85 cloning and sequence analyses. Given the potential immunological importance of TLRs against fungal pathogens, we hypothesized that TLRs would be subjected to purifying selection in species which show marked resistance to $\mathrm{Bd}$. In addition, we also conducted preliminary investigation of TLR expression using published transcriptomic data, to determine whether basal expression is similar across different life stages and tissue types.

\section{Methods}

Animals

All sample collection was approved by Hiroshima University Animal Research Committee, approval number G14-2. Adult skin tissues were collected from three common Ranidae frog species from Japan $(n=5$ per species): the Japanese brown frog (Rana japonica), the montane brown frog (Rana ornativentris), and Tago's brown frog (Rana tagoi tagoi). All frogs are the same individuals used previously to characterize MHC class I and II genes (Lau et al., 2016, 2017, Table S1). All animals were housed in laboratory conditions for a minimum of five weeks and exhibited no clinical signs of disease prior to euthanasia, and thus considered 'healthy'. Animals were euthanized through immersion in tricaine methanesulfonate (MS222, 0.5-3 g/L water), and preserved in RNAlater (Applied Biosystems, Carlsbad, CA, USA) at $-20^{\circ} \mathrm{C}$ prior to excision of skin sample and genomic DNA (gDNA) extraction using DNAsuisui-F (Rizo Inc., Tsukuba, Japan) following manufacturer's protocol. 
102

103

104

105

106

107

108

109

110

111

112

113

114

115

116

117

\section{Isolation of TLR genes from transcriptome data set and primer design}

To isolate TLR2 and TLR4 genes, we utilised the published transcriptomic data set previously compiled using Illumina sequences from immune tissues of the three species (Lau et al., 2017). Briefly, we used the assembled transcripts that were annotated with NCBI-BLAST-2.3.30 against the Swissprot protein database (httpr://www.expasy.ch/sprot), and isolated all transcripts that had top hits from BLAST search to published TLR genes of other vertebrates. We manually scanned the NCBI-BLAST search results and aligned each transcript with orthologous genes from Xenopus laevis and Nanorana parkeri (Xenbase, http://www.xenbase.org/, RRID:SCR_003280, and GenBank accession numbers XM_002933491, XM_018557931, XM_018232906, XM_018565865). Due to low coverage of sequence data, full-length contigs were not available for TLR2 and TLR4 genes in all three species (Figures S1 and S2); in such cases, fragmented contigs were used in the alignment. The genomic structure of most vertebrate TLR genes are unique in that the majority of their coding sequence is located within a single exon. From the alignments, we used Primer 3 (Rozen \& Skaletsky, 1998) to design degenerate primers that amplified 2348 bp and 2072 bp fragments within a single exon of TLR2 (RanaTLR2_F: 5'-TGRTTGCATACATATGGAGTTG-3', RanaTLR2_R: 5'GTGGTCCTCTGGCTGAAGAG-3') and TLR4 (RanaTLR4_F: 5'-CTGGCAAGCCTTTCTGAACT-3', RanaTLR4_R: 5'AGCGGARCATCAACTTTACG-3'), respectively, across all three species (Table S1).

\section{TLR PCR and sequencing}

Polymerase chain reaction (PCR) amplification was conducted in Applied Biosystems ${ }^{\circledR}$ Veriti $^{\circledR}$ thermal cycler in $10 \mu \mathrm{L}$ reactions with $0.25 \mathrm{U}$ TaKaRa Ex Taq ${ }^{\circledR}$ polymerase (Takara Bio Inc.), 1x Ex Taq PCR buffer, $0.2 \mathrm{mM}$ each dNTP, and $0.7 \mu \mathrm{M}$ each primer and $0.5-1.0 \mu \mathrm{L}$ skin gDNA samples ( $\mathrm{n}=5$ per species) with the following cycle condition: initial Taq activation at $95^{\circ} \mathrm{C}$ for $1 \mathrm{~min}$, then 35 cycles of 30 -s denaturation at $95^{\circ} \mathrm{C}, 30-\mathrm{s}$ annealing at $60^{\circ} \mathrm{C}(T L R 2)$ or $61^{\circ} \mathrm{C}(T L R 4)$, and 80 -s extension at $72^{\circ} \mathrm{C}$, then a final extension of $72^{\circ} \mathrm{C}$ for 3 min. Since TLR alleles could not be phased in heterozygous individuals by sequencing alone, we used molecular cloning following by Sanger sequencing. PCR products were ligated into T-Vector pMD20 (Takara Bio Inc) 
126

127

128

131

132

133

134

135

136

137

138

139

140

141

142

143

144

145

146

147

using DNA Ligation Kit 2.1 (Takara Bio Inc.) and incubated for $30 \mathrm{~min}$ at $16^{\circ} \mathrm{C}$. For cloning, ligation reactions were transformed into JM109 competent cells (Takara Bio Inc.) and cultured on selective LB plates containing $50 \mu \mathrm{g} / \mathrm{mL}$ ampicillin overnight at $37^{\circ} \mathrm{C}$. We then amplified positive clones ( $4-10$ per individual reaction) using M13 primers and similar PCR conditions, and purified using ExoSAP-IT® ${ }^{\circledR}$ (Affymetric Inc., Santa Clara, USA). As amplicons were over $2 \mathrm{kbp}$ length, we utilised four to six additional sequencing primers (Table S2, Figures S3 and S4) in addition to M13 primers for sequencing with BigDye ${ }^{\circledR}$ Terminator Cycle Sequencing kit (Applied Biosystems, Foster City, USA) and ABI 3130xl automated sequencer.

Sequence analyses, $d_{N} / d_{S}$ comparison with other genes, and selection tests

We measured polymorphism and divergence of the TLR2 and TLR4 sequences using DnaSP 6.10.03 (Rozas et al., 2017), including number of segregating sites (S), number of alleles $\left(N_{A}\right)$, average number of nucleotide differences (k), nucleotide diversity $(\pi)$, Tajima's D (D) and normalized Fay and Wu's H (Hn). We calculated synonymous $\left(d_{S}\right)$ and nonsynonymous $\left(d_{N}\right)$ divergence and the ratio $\left(d_{N} / d_{s}\right)$ between the focal species using MEGA7 (Kumar, Stecher \& Tamura, 2016). We then compared the $d_{N} / d_{s}$ ratio with that of major histocompatibility complex (MHC) class I and II (average among the three species), which are known to be under balancing selection in these species (Lau et al., 2016, 2017). In addition, we compared with $d_{N} / d_{s}$ ratio of all orthologous genes collated from the transcriptome data set of Lau et al (2017). This consisted of over 3000 orthologous amino acid sequences from each of the three species that were identified using Proteinortho V5.15 (Lechner et al., 2011). We then extracted nucleotide coding sequences of orthologous genes from transcriptome data sets using a custom python script, and used PhyloTreePruner (Kocot et al., 2013) to align the sequences and remove paralogues. Finally, all sequences were compiled together and maximum likelihood estimates of $\omega\left(d_{N} / d_{S}\right)$ were calculated using CODEML in PAML 4.9 (Yang, 2007).

To test for selection, we used McDonald-Kreitman (MK) test in DnaSP 6.10.03 to compare species-wide data with outgroup sequences from distantly related Ranidae frogs (Odorrana amamiensis and O. ishikawae, transcriptome data, source: T. Igawa, GenBank accession numbers MH165314-MH165317). In addition, we 
150 tested for sequence-wide neutral $\left(d_{N}=d_{S}\right)$, purifying $\left(d_{N}<d_{S}\right)$ and positive $\left(d_{N}>d_{S}\right)$ selection using codon-

151 based Z tests with 1000 bootstrap replicates in MEGA7. To infer specific codons as positively selected sites

152 (PSSs) with $\omega\left(d_{N} / d_{s}\right)>1$, we used omegaMap version 5.0 (Wilson \& McVean, 2006) to perform Bayesian

153 inference on independent alignments for each species and gene, following Lau et al. (2016). Neighbour-

154 joining phylogenetic trees from amino acid alignments ( $p$-distance) were constructed independently for TLR2

155 and TLR4 in MEGA7. Protein domain structures of TLR2 and TLR4 were predicted using SMART (Letunic,

156 Doerks \& Bork, 2015).

157

158

\section{Expression of TLRs}

In order to investigate baseline expression of our candidate TLRs, we extracted expression levels from our transcriptome data set (Lau et al., 2017). RSEM v 1.3.0 (Li \& Dewey, 2011) was used to extract trimmed mean log expression ratio, or TMM-normalized values which represent estimated relative RNA production levels (Robinson \& Oshlack, 2010), for TLR2 and TLR4 transcripts in each of the 12 samples (Table S1). We compared TMM-normalized values within adults (blood, skin and spleen), using false discovery rate (FDR) cut-off of 0.001, to determine whether expression was ubiquitous. In addition, we checked if expression in tadpoles was different to adults, using stage 24 and stage 29 tadpoles (in R. japonica and R.ornativentris) (Gosner, 1960). In the cases where full-length contigs were not available from low sequence coverage ( $R$. ornativentris TLR2 and TLR4, and R. t. tagoi TLR4), we obtained expression values for each fragmented contig.

\section{Results}

We characterized a total of 27 TLR2 and 20 TLR4 alleles from the three study species, all of which were distinctive at the amino-acid level. Rana japonica had seven TLR2 and five TLR4 alleles, $R$. ornativentris had ten TLR2 and seven TLR4 alleles, and $R$. tagoi tagoi had ten TLR2 and eight TLR4 alleles (Table 1, Figures S3 and S4, GenBank accession numbers MG999527-MG999573). All alleles clustered phylogenetically into species-specific clades (Figure 1) and had similar domain structure to that of other frogs (Figure S5). Allelic 
173 diversity among species was high (Table 1, Table S3), especially TLR2 in R. ornativentris and $R$. t. tagoi, where

174 all individuals were heterozygous with two unique alleles.

175 Selection tests over the entire alignment indicated that TLR2 and TLR4 in the three focal species are under

176 purifying selection (Z-value $=2.66-3.83, \mathrm{p}<0.01$, Table 2$)$ with the exception of TLR4 in $R . t$. tagoi $(Z$-value $=$

177 1.26, $p=0.102)$. In addition, Tajima's D, normalized Fay and Wu's $H$, and the McDonald-Kreitman test

178 showed no significant support for selection (Table 1). However, omegaMap analyses identified six to nine

179 positively selected sites (PSSs) in either TLR2 or TLR4 of each of the three focal species (Posterior probability

$180>99 \%)$. Of these, two (22.2\%) to six (75.0\%) PSSs were located in predicted leucine rich repeat domains

181 (Table 2, Figure S3 and S4). There were no PSSs common in all three species studied, but two PSSs of TLR4

182 (sites D56 and S65) were shared across two species (Table 2, Figure S4).

183 Pairwise comparisons of $d_{N} / d_{s}$ ratios between Rana species were low and ranged from 0.188 to 0.398 (Table

184 3). These ratios were comparable to that of MHC class $1 \alpha 3$ and MHC class II $\beta 2$ domains as well as other

185 transcribed genes within the species (mean $d_{N} / d_{S}$ of over 3000 genes $=0.380$, Figure 2 ). In contrast, these

186 ratios were lower than that of $\mathrm{MHC}$ class I $\alpha 1$ and $\alpha 2$ domains and class II $\beta 1$ domain.

187 When we checked published transcriptome data for TLR2 and TLR4 expression, preliminary examination of

188 adult tissues indicated no significant differences between tissue types (blood, spleen, skin) at FDR cut-off of

189 0.0001. Nevertheless, overall expression of TLR2 and TLR4 was low and there were a few consistent trends

190 seen across all three species. This included higher expression of TLR2 in adult skin relative to blood, and

191 lower expression of TLR4 in adult skin relative to either blood or spleen (Table 4). Across life stages,

192 expression of TLR2 and TLR4 was seemingly low in tadpoles relative to adult samples (FDR $>0.0001$, n.s.,

193 Table 4).

194 Discussion 
195

196

197

198

200

201

202

203

204

205

206

207

208

209

210

211

212

213

214

215

216

217

218

The preliminary characterization of TLR2 and TLR4 in the three focal species here provides a platform for future population genetics studies across the species' distributions, uncovering the full TLR diversity with more targeted PCR and sequencing approaches. Overall allelic diversity of TLR2 and TLR4 appeared to be high, whereby only a few alleles were shared between more than one individual (Table S3). Commonly shared alleles were more apparent in $R$. japonica, likely due to a population bottleneck history in the source population of Etajima (Lau et al., 2016). The presence of the TLR4 gene in amphibians was previously unclear, described as 'putative' in Xenopus frogs (Ishii et al., 2007) and 'predicted' from genomic data in Lithobates catesbeianus and Nanorana parkeri (GenBank accessions XP_018421367 and PIO23183; Sun et al., 2015; Hammond et al., 2017), and undetected in newts (Babik et al., 2014). The characterization of TLR4 genes in this study supports the existence of this gene family in anurans, whereby TLR4 alleles of the three Rana species were similar in phylogeny and domain structure to that of other frogs.

From selection tests, we found overall evidence of purifying selection and no support for sequence-wide positive selection. This agrees with data from other vertebrates, including newts, where TLRs are regarded as conserved with their evolution predominated by purifying selection (Roach et al., 2005; Babik et al., 2014).

The $d_{N} / d_{S}$ ratios in TLR2 and TLR4 of the Rana species studied here were remarkably low compared to that of external domains of MHC class I ( $\alpha 1$ and $\alpha 2$ domains) and class II ( $\beta 1$ domain), which are considered to be under balancing selection. However, the low $d_{N} / d_{S}$ of TLR2 and TLR4 was comparable to $d_{N} / d_{S}$ of MHC class 1 $\alpha 3$ and $\mathrm{MHC}$ class II $\beta 2$ domains which are intracellular or not involved in peptide recognition, as well as those of over 3000 transcribed genes within the species. These findings further support that TLR2 and TLR4 are under functional constraint.

Although we found that most of the TLR2 and TLR4 sequences of the Japanese Rana frogs were evolutionarily constrained, we identified evidence of adaptive evolution occurring at individual codon sites in our alignment, similar to other vertebrates studied (Wlasiuk \& Nachman, 2010; Shang et al., 2018). When comparing with codon sites predicted to be important for binding of non-fungal ligands (Figures S3 and S4), two PSS 
219 identified in R.ornativentris TLR2 (Q284 and V299) corresponded to sites in human TLR2 predicted to be

220 involved in ligand binding of lipopeptides (N294 and L312, Jin et al., 2007). In addition, one PSS each of $R$.

221 tagoi tagoi (T128) and R. ornativentris (Q253) corresponded to human TLR4 sites predicted to be involved in

222 secondary (N268) and phosphate (K388) binding, respectively, of bacterial lipopolysaccharides (Park et al.,

223 2009). Positive selection at identical codon sites across different species, as observed in MHC adaptive

224 immune genes (Lau et al., 2016, 2017), could be driven by a single selective force that is pathogen-related.

225 However, in Rana TLRs there were no PSSs shared across all three species studied. As we did not examine the

226 patterns of selection in species which are susceptible to Bd, any link is currently speculative; nevertheless, we

227 cannot rule out the possibility of adaptive evolution, potentially driven by pathogens such as Bd, acting on

228 TLR of the study species in recent evolutionary history.

229 Preliminary examination of TLR2 and TLR4 expression levels extracted from transcriptome data showed

230 overall low expression. While the expression data is derived from single individuals that were housed in

231 disease-free environments, it appears that skin of healthy frogs that are not immune-challenged express TLR2

232 more so than TLR4. However, expression of immune-related genes could be modulated following immune or

233 stress challenges, and future studies should monitor immune gene expression following experimental

234 infection with pathogens like $\mathrm{Bd}$. The adult tissue-specific differences in TLR2 and TLR4 expression from $R$.

235 ornativentris in this study were distinct from B. maxima (Zhao et al., 2014), but sample size should be

236 increased for both species before further inferences can be made. A previous study in Xenopus frogs

237 detected ubiquitous expression of both TLR2 and TLR4 in adults and tadpoles using PCR, but expression levels

238 were not quantified (Ishii et al., 2007). Although we found low TLR expression in tadpoles in this study,

239 further conclusions cannot be made due to limited sampling and overall low TLR expression across the

240 samples. Future quantitative studies can investigate expression level changes of TLRs during development

241 from tadpole to adults, as well as that of other innate and adaptive immune genes extracted from the

242 transcriptome data set (Lau et al., 2017). 
244 In this study, we characterized TLR2 and TLR4 genes from three Japanese Rana species. We provide strong

245 evidence of purifying selection acting across the TLR2 and TLR4, and evidence of a few specific codon sites

under positive selection. Further research is necessary to determine if the positive selection we detected is

247

due to pathogen-driven selection. Since immunity to infectious diseases is usually polygenetic, our study adds

to the growing body of literature related to genes that potentially impact resistance to Bd and other pathogens in amphibians.

\section{References}

Babik W., Dudek K., Fijarczyk A., Pabijan M., Stuglik M., Szkotak R., Zielinski P. 2014. Constraint and adaptation in newt Toll-like receptor genes. Genome Biology and Evolution 7:81-95.

Bataille A., Fong JJ., Cha M., Wogan GOU., Baek HJ., Lee H., Min MS., Waldman B. 2013. Genetic evidence for a high diversity and wide distribution of endemic strains of the pathogenic chytrid fungus Batrachochytrium dendrobatidis in wild Asian amphibians. Molecular Ecology 22:4196-4209.

Daszak P., Cunningham AA., Hyatt AD. 2003. Infectious disease and amphibian population declines. Diversity and Distributions 9:141-150.

Fisher MC. 2009. Endemic and introduced haplotypes of Batrachochytrium dendrobatidis in Japanese amphibians: Sink or source? Molecular Ecology 18:4731-4733.

Goka K., Yokoyama J., Une Y., Kuroki T., Suzuki K., Nakahara M., Kobayashi A., Inaba S., Mizutani T., Hyatt AD. 2009. Amphibian chytridiomycosis in Japan: Distribution, haplotypes and possible route of entry into Japan. Molecular Ecology 18:4757-4774.

Gosner KL. 1960. A simplified table for staging anuran embryos larvae with notes on identification. Herpetologica 16:183-190.

Hammond SA., Warren RL., Vandervalk BP., Kucuk E., Khan H., Gibb EA., Pandoh P., Kirk H., Zhao Y., Jones M., Mungall AJ., Coope R., Pleasance S., Moore RA., Holt RA., Round JM., Ohora S., Walle B V., Veldhoen N., Helbing CC., Birol I. 2017. The North American bullfrog draft genome provides insight into hormonal regulation of long noncoding RNA. Nature Communications 8:1433.

Ishii A., Kawasaki M., Matsumoto M., Tochinai S., Seya T. 2007. Phylogenetic and expression analysis of amphibian Xenopus Toll-like receptors. Immunogenetics 59:281-293.

Jin MS., Kim SE., Heo JY., Lee ME., Kim HM., Paik SG., Lee H., Lee JO. 2007. Crystal structure of the TLR1-TLR2 heterodimer induced by binding of a tri-acylated lipopeptide. Cell 130:1071-1082.

Kocot KM., Citarella MR., Moroz LL., Halanych KM. 2013. PhyloTreePruner: A phylogenetic tree-based approach for selection of orthologous sequences for phylogenomics. Evolutionary Bioinformatics 2013:429-435.

Kumar S., Stecher G., Tamura K. 2016. MEGA7: Molecular Evolutionary Genetics Analysis version 7.0 for bigger datasets. Molecular Biology and Evolution 33:1870-1874. 
277

282

283

284

285

286

287

288

289

290

291

292

293

294

295

296

297

298

299

300

301

302

303

304

305

306

307

308

309

310

311

312

313

314

315

Lau Q., Igawa T., Komaki S., Satta Y. 2016. Characterisation of major histocompatibility complex class I genes in Japanese Ranidae frogs. Immunogenetics 68:797-806.

Lau Q., Igawa T., Minei R., Kosch TA., Satta Y. 2017. Transcriptome analyses of immune tissues from three Japanese frogs (genus Rana) reveals their utility in characterizing major histocompatibility complex class II. BMC Genomics 18:994.

Lechner M., Findeiß S., Steiner L., Marz M., Stadler PF., Prohaska SJ. 2011. Proteinortho: Detection of (Colorthologs in large-scale analysis. BMC Bioinformatics 12:124.

Letunic I., Doerks T., Bork P. 2015. SMART: Recent updates, new developments and status in 2015. Nucleic Acids Research 43:D257-D260.

Li B., Dewey CN. 2011. RSEM: accurate transcript quantification from RNA-Seq data with or without a reference genome. BMC bioinformatics 12:323.

Longcore J., Longcore J., Pessier A., Halteman WA. 2007. Chytridiomycosis widespread in anurans of northeastern United States. Journal of Wildlife Management 71:435-444.

Luther K., Ebel F. 2006. Toll-like receptors: Recent advances, open questions and implications for aspergillosis control. Medical Mycology 44:219-227.

Medzhitov R. 2001. Toll-like receptors and innate immunity. Nature Reviews Immunology 1:135-145.

Mikami T., Miyashita H., Takatsuka S., Kuroki Y., Matsushima N. 2012. Molecular evolution of vertebrate Toll-like receptors: Evolutionary rate difference between their leucine-rich repeats and their TIR domains. Gene 503:235-243.

Noreen M., Arshad M. 2015. Association of TLR1, TLR2, TLR4, TLR6, and TIRAP polymorphisms with disease susceptibility. Immunologic Research 62:234-252.

Park BS., Song DH., Kim HM., Choi BS., Lee H., Lee JO. 2009. The structural basis of lipopolysaccharide recognition by the TLR4-MD-2 complex. Nature 458:1191-1195.

Poltorak A., He X., Smirnova I., Liu M., Huffel C Van., Du X., Birdwell D., Alejos E., Silva M., Galanos C., Freudenberg M., Ricciardi-castagnoli P., Layton B., Beutler B. 1998. Defective LPS Signaling in C3H / HeJ and C57BL / 10ScCr Mice : Mutations in TLR4 Gene. Science. 282:2085-2088.

Quach H., Wilson D., Laval G., Patin E., Manry J., Guibert J., Barreiro LB., Nerrienet E., Verschoor E., Gessain A., Przeworski M., Quintana-Murci L. 2013. Different selective pressures shape the evolution of toll-like receptors in human and African great ape populations. Human Molecular Genetics 22:4829-4840.

Richmond JQ., Savage AE., Zamudio KR., Rosenblum EB. 2009. Toward immunogenetic studies of amphibian chytridiomycosis: linking innate and acquired immunity. BioScience 59:311-320.

Roach JC., Glusman G., Rowen L., Kaur A., Purcell MK., Smith KD., Hood LE., Aderem A. 2005. The evolution of vertebrate Toll-like receptors. Proceedings of the National Academy of Sciences of the United States of America 102:9577-9582.

Robinson MD., Oshlack A. 2010. A scaling normalization method for differential expression analysis of RNA-seq data. Genome Biology 11:R25.

Roeder A., Kirschning CJ., Rupec RA., Schaller M., Weindl G., Korting HC. 2004. Toll-like receptors as key mediators in innate antifungal immunity. Medical Mycology 42:485-498.

Rozas J., Ferrer-Mata A., Sánchez-DelBarrio JC., Guirao-Rico S., Librado P., Ramos-Onsins SE., Sánchez-Gracia A. 
316

317

318

319

320

321

322

323

324

325

326

327

328

329

330

331

332

333

334

335

336

337

338

339

340

341

342

343

344

345

346

347

348

349

350

351

2017. DnaSP 6: DNA sequence polymorphism analysis of large data sets. Molecular Biology and Evolution 34:3299-3302.

Rozen S., Skaletsky HJ. 1998. Primer3. In: Bioinformatics Methods and Protocols Methods in Molecular Biology. 141.

Shang S., Zhong H., Wu X., Wei Q., Zhang H., Chen J., Chen Y., Tang X., Zhang H. 2018. Genomic evidence of gene duplication and adaptive evolution of Toll like receptors (TLR2 and TLR4) in reptiles. International Journal of Biological Macromolecules 109:698-703.

Skerratt LF., Berger L., Speare R., Cashins S., McDonald KR., Phillott AD., Hines HB., Kenyon N. 2007. Spread of chytridiomycosis has caused the rapid global decline and extinction of frogs. EcoHealth 4:125-134.

Smirnova I., Hamblin MT., McBride C., Beutler B., Rienzo A Di. 2001. Excess of rare amino acid polymorphisms in the toll-like receptor 4 in humans. Genetics 158:1657-1664.

Smirnova I., Mann N., Dols A., Derkx HH., Hibberd ML., Levin M., Beutler B. 2003. Assay of locus-specific genetic load implicates rare Toll-like receptor 4 mutations in meningococcal susceptibility. Proceedings of the National Academy of Sciences of the United States of America 100:6075-80.

Sun Y-B., Xiong Z-J., Xiang X-Y., Liu S-P., Zhou W-W., Tu X-L., Zhong L., Wang L., Wu D-D., Zhang B-L., Zhu C-L., Yang M-M., Chen H-M., Li F., Zhou L., Feng S-H., Huang C., Zhang G-J., Irwin D., Hillis DM., Murphy RW., Yang H-M., Che J., Wang J., Zhang Y-P. 2015. Whole-genome sequence of the Tibetan frog Nanorana parkeri and the comparative evolution of tetrapod genomes. Proceedings of the National Academy of Sciences 112:E1257E1262.

Takeda K., Kaisho T., Akira S. 2003. Toll-Like Receptors. Annual Review of Immunology 21:335-376.

Tschirren B., Andersson M., Scherman K., Westerdahl H., Mittl PRE., Raberg L. 2013. Polymorphisms at the innate immune receptor TLR2 are associated with Borrelia infection in a wild rodent population. Proceedings of the Royal Society B: Biological Sciences 280:20130364-20130364.

Voogdt CGP., Bouwman LI., Kik MJL., Wagenaar JA., van Putten JPM. 2016. Reptile Toll-like receptor 5 unveils adaptive evolution of bacterial flagellin recognition. Scientific Reports 6:19046.

Wake DB., Vredenburg VT. 2008. Are we in the midst of the sixth mass extinction? A view from the world of amphibians. Proceedings of the National Academy of Sciences of the United States of America 105:1146611473.

Wilson DJ., McVean G. 2006. Estimating diversifying selection and functional constraint in the presence of recombination. Genetics 172:1411-1425.

Wlasiuk G., Nachman MW. 2010. Adaptation and constraint at toll-like receptors in primates. Molecular Biology and Evolution 27:2172-2186.

Yang Z. 2007. PAML 4: phylogenetic analysis by maximum likelihood. Molecular Biology and Evolution 24:15861591.

Zhao F., Yan C., Wang X., Yang Y., Wang G., Lee W., Xiang Y., Zhang Y. 2014. Comprehensive transcriptome profiling and functional analysis of the frog (Bombina maxima) immune system. DNA Research 21:1-13. 


\section{Table $\mathbf{1}$ (on next page)}

Polymorphism of TLR2 and TLR4

Polymorphism of TLR2 and TLR4 in R. japonica, R. ornativentris and R. tagoi tagoi. 
1 Table 1. Polymorphism of TLR2 and TLR4 in R. japonica, R. ornativentris and R. tagoi tagoi.

\begin{tabular}{lccccccccc}
\hline Gene & $2 \mathrm{~N}$ & nsites & $\mathrm{N}_{\mathrm{A}}$ & $\mathrm{S}$ & $\mathrm{k}$ & $\pi$ & $\mathrm{D}$ & $\mathrm{Hn}$ & $\begin{array}{l}\text { MK P- } \\
\text { value }\end{array}$ \\
\hline TLR2 & & & & & & & & & \\
R. japonica & 10 & 2312 & 7 & 28 & 10.48 & 0.0045 & -0.474 & -0.873 & 0.629 \\
$\begin{array}{l}\text { R. ornativentris } \\
\text { R. tagoi tagoi }\end{array}$ & 10 & 2312 & 10 & 44 & 13.89 & 0.0060 & -0.521 & -0.242 & 1.000 \\
TLR4 & 2312 & 10 & 39 & 13.29 & 0.0058 & -0.292 & -1.108 & 0.215 \\
R. japonica & 10 & 2072 & 5 & 41 & 19.20 & 0.0093 & -0.183 & -0.144 & 0.279 \\
R. ornativentris & 10 & 2078 & 7 & 35 & 14.71 & 0.0071 & 0.008 & -0.510 & 0.266 \\
R. tagoi tagoi & 10 & 2072 & 8 & 25 & 8.57 & 0.0041 & -0.584 & -1.539 & 0.127
\end{tabular}

2 2N: number of gene copies studied; nsites: nucleotide length of sequence; NA: number of alleles; $\mathrm{S}$ : number of segregating sites; $\mathrm{k}$ : average number of nucleotide

3 differences; $\pi$ : nucleotide diversity; D: Tajima's $D$ value for all sites (no values were significant at $p<0.01$ ); $\mathrm{Hn}$ : Fay and Wu's normalized $H$ value for all sites (no

values significant $\mathrm{p}<0.01$ ); MK P-value: McDonald and Kreitman Fisher's exact test P-value

5 


\section{Table 2 (on next page)}

Codon-based $Z$ tests for global selection

Codon-based $Z$ tests for global selection (Z statistics), and specific codon sites under positive selection detected by omegaMap. Codon sites in identical positions in more than one species are underlined. 
1 Table 2. Codon-based Z tests for global selection (Z statistics), and specific codon sites under positive 2 selection detected by omegaMap. Codon sites in identical positions in more than one species are 3 underlined.

\begin{tabular}{lllll}
\hline Gene & Neutrality & Purifying & Positive & Positively selected sites (PSS) \\
\hline TLR2 & & & & \\
$\begin{array}{l}\text { R. japonica } \\
\text { R. ornativentris }\end{array}$ & $-2.72^{*}$ & $2.69^{*}$ & -2.63 n.s. & $12,23,95^{\#}, 164,428^{\#}, 672$ \\
$\begin{array}{l}\text { R. tagoi tagoi } \\
\text { TLR4 }\end{array}$ & $-3.81^{* *}$ & $3.66^{*}$ & -2.77 n.s. & $11,53^{\#}, 75^{\#}, 207,284,299,417,509,535$ \\
R. japonica & & -3.76 n.s. & $105^{\#}, 192,235,265,407,458^{\#}, 485^{\#}, 486^{\#}$ \\
R. ornativentris & $-3.16^{*}$ & $3.11^{*}$ & -3.11 n.s. & $12,46^{\#}, \underline{65^{\#}}, 129,133,221,339,430^{\#}$ \\
R. tagoi tagoi & $-3.34^{*}$ & $3.34^{* *}$ & -3.32 n.s. & $35^{\#}, \underline{56^{\#}}, 77^{\#}, 173,253^{\#}, 378^{\#}, 481^{\#}, 691$ \\
\hline
\end{tabular}

${ }^{*} p<0.01,{ }^{* *} p<0.001$, n.s. $-p>0.05$; \#PS located in leucine rich region (LRR)

5 


\section{Table 3 (on next page)}

dN and dS of TLR2, TLR4 and MHC genes

Nonsynonymous $\left(d_{N}\right)$ and synonymous $\left(d_{s}\right)$ divergence between the three focal Rana species, for TLR2 and TLR4 genes as well as previously characterized MHC class I and II loci from these three species. 
1 Table 3. Nonsynonymous $\left(d_{N}\right)$ and synonymous $\left(d_{s}\right)$ divergence between the three focal Rana species, 2 for TLR2 and TLR4 genes as well as previously characterized MHC class I and II loci from these three 3 species.

\begin{tabular}{llll}
\hline Gene & $\mathrm{d}_{\mathrm{N}}$ & $\mathrm{d}_{\mathrm{s}}$ & $\mathrm{d}_{\mathrm{N}} / \mathrm{d}_{\mathrm{s}}$ \\
\hline TLR2 & & & \\
Rj-Ro & 0.014 & 0.077 & 0.188 \\
Rj-Rt & 0.015 & 0.069 & 0.213 \\
Ro-Rt & 0.020 & 0.065 & 0.316 \\
$\quad$ all three species & & & 0.291 \\
TLR4 & & & \\
Rj-Ro & 0.016 & 0.078 & 0.207 \\
Rj-Rt & 0.018 & 0.062 & 0.293 \\
Ro-Rt & 0.020 & 0.051 & 0.398 \\
all three species & & & 0.379 \\
MHC class I $\alpha 1$ & & & 1.632 \\
MHC class I $\alpha 2$ & 0.092 & 0.056 & 1.525 \\
MHC class I $\alpha 3$ & 0.082 & 0.054 & \\
MHC class II $\beta 1$ & 0.035 & 0.073 & 0.476 \\
MHC class II $\beta 2$ & & & \\
\hline
\end{tabular}

4 


\section{Table 4 (on next page)}

Expression of TLR2 and TLR4 across different tissues and life stages based on transcriptome data

Normalized expression of TLR2 and TLR4 across different tissues and life stages based on transcriptome data set of Lau et al. (2017). 
1 Table 4. Normalized expression of TLR2 and TLR4 across different tissues and life stages based on

2 transcriptome data set of Lau et al. (2017).

\begin{tabular}{|c|c|c|c|c|c|c|c|}
\hline Gene & Species & $\begin{array}{l}\text { Transcript } \\
\text { length (bp) }\end{array}$ & $\begin{array}{l}\text { Adult } \\
\text { blood }\end{array}$ & $\begin{array}{l}\text { Adult } \\
\text { skin }\end{array}$ & $\begin{array}{l}\text { Adult } \\
\text { spleen }\end{array}$ & $\begin{array}{l}\text { S24 } \\
\text { tadpole }\end{array}$ & $\begin{array}{l}\text { s29 } \\
\text { tadpole }\end{array}$ \\
\hline \multirow[t]{5}{*}{ TLR2 } & R.japonica & 3285 & 0.25 & 1.37 & 2.60 & - & 0.05 \\
\hline & R. ornativentris & & & & & & \\
\hline & fragment 1 & 1417 & 0 & 0.73 & 0.11 & 0.20 & 0 \\
\hline & fragment 2 & 1324 & 0.16 & 0.88 & 0.22 & 0.25 & 0 \\
\hline & R.t. tagoi & 2686 & 0.86 & 2.55 & 1.59 & - & - \\
\hline \multirow[t]{8}{*}{ TLR4 } & R.japonica & 2830 & 0.20 & 0.03 & 1.14 & - & 0.01 \\
\hline & R. ornativentris & & & & & & \\
\hline & fragment 1 & 444 & 0.16 & 0 & 0.32 & 0 & 0 \\
\hline & fragment 2 & 548 & 0 & 0 & 0.55 & 0 & 0.52 \\
\hline & fragment 3 & 405 & 0 & 0.28 & 0.58 & 0 & 0 \\
\hline & R.t. tagoi & & & & & & \\
\hline & fragment 1 & 1454 & 0.11 & 0 & 0.50 & - & - \\
\hline & fragment 2 & 629 & 0.18 & 0 & 1.21 & & \\
\hline
\end{tabular}

3 
Figure 1

Phylogenetic relationships of TLR2 and TLR4 alleles from three Japanese Rana species.

Phylogenetic relationships of (A) TLR2 and (B) TLR4 alleles identified in $R$. japonica (red), $R$. ornativentris (blue) and $R$. tagoi tagoi (orange) and other species based on amino acid alignments (neighbour-joining method). Human TLR5 and TLR6 were used as outgroup sequences. Accession numbers for sequences are indicated. Sequences obtained from transcriptome data include: Lithobates catesbeianus (DRA accession number SRP051787), and Odorrana amamiensis and O. ishikawae (GenBank accessions MH165314-MH165317). Image sources: Q. Lau.

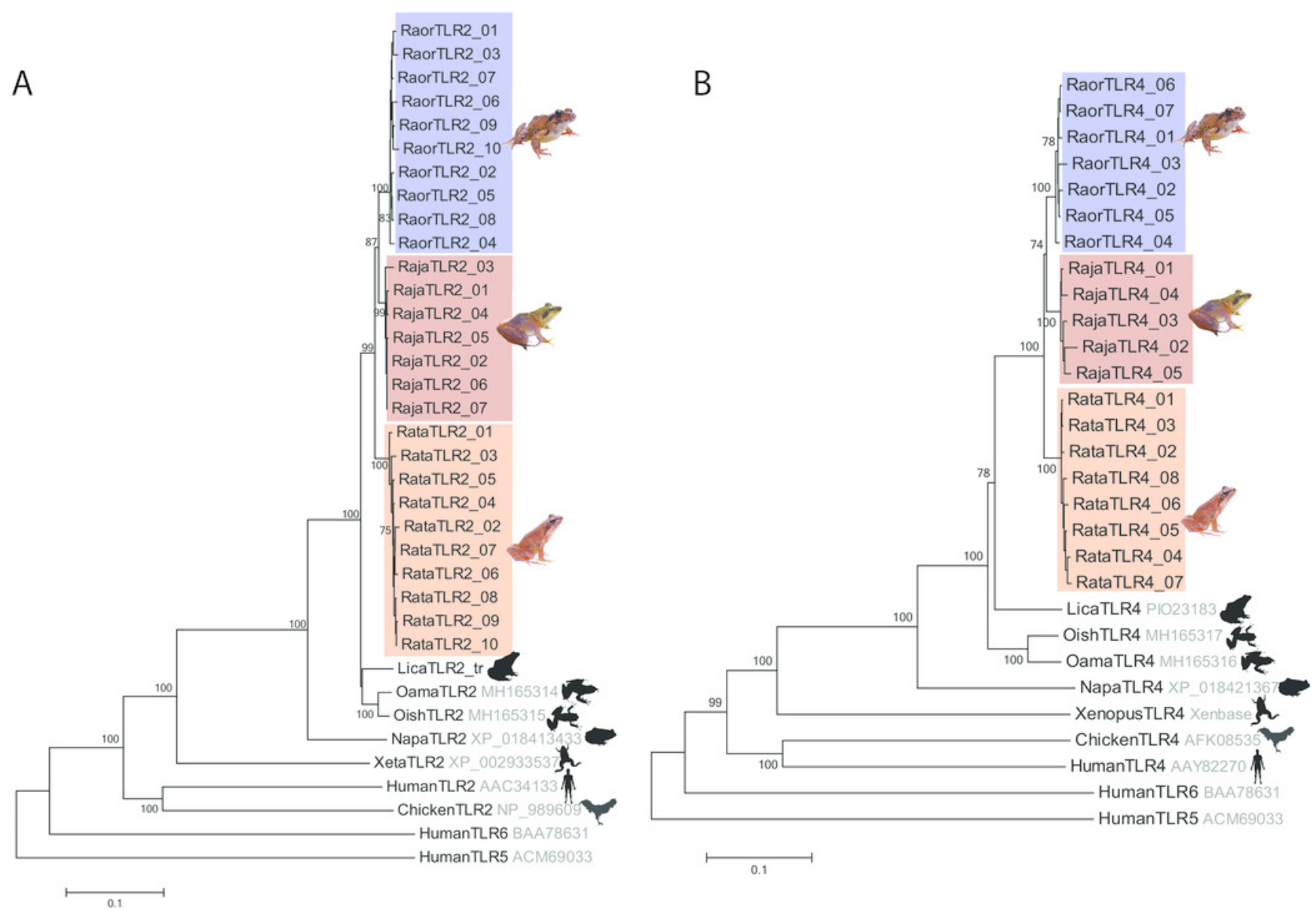




\section{Figure 2 (on next page)}

Distribution of $d_{N} / d_{S}$ ratios from over 3000 genes isolated from transcriptome data compared to TLR and MHC.

Distribution of $\mathrm{d}_{\mathrm{N}} / \mathrm{d}_{\mathrm{S}}$ ratios from over 3000 genes isolated from transcriptome data of $R$. japonica, $R$. ornativentris and $R$. tagoi tagoi. $\mathrm{d}_{\mathrm{N}} / \mathrm{d}_{\mathrm{S}}$ location of TLR2 and TLR4, as well as MHC class I and II domains are marked with arrows. 


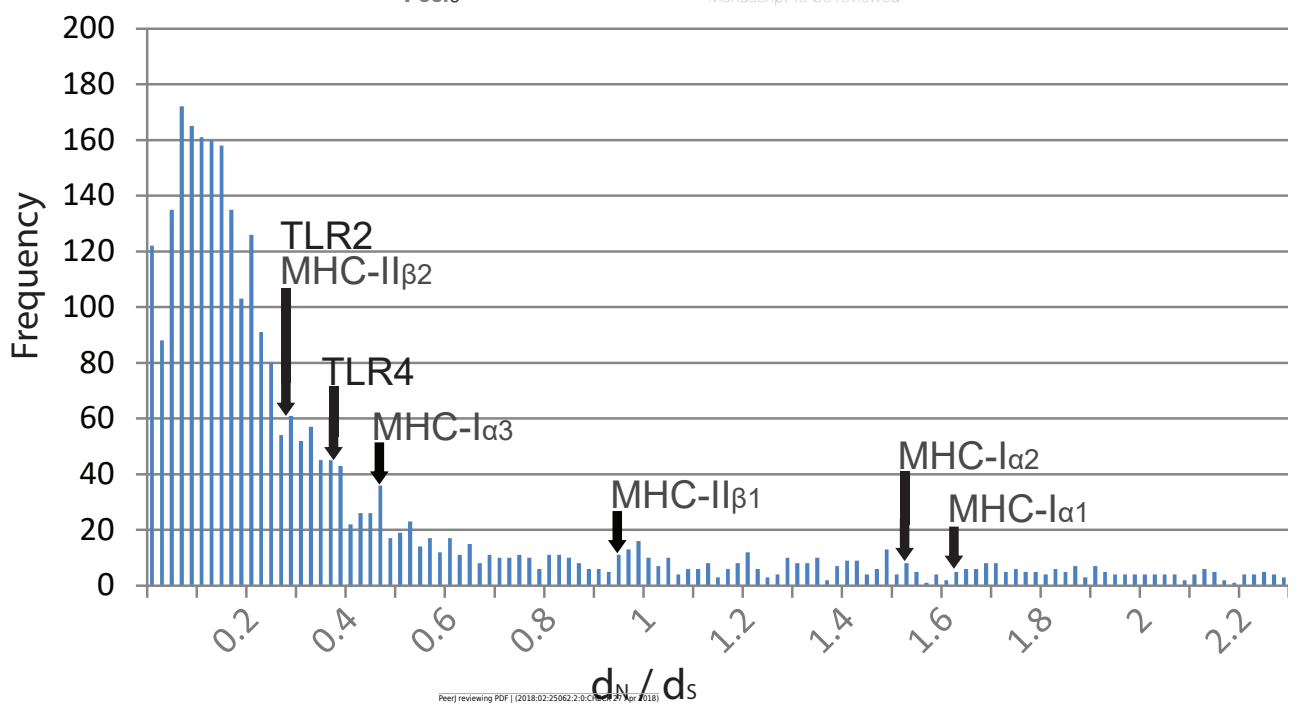

